\title{
Characterization of Sticking Residue on Tablet Punch Faces by Scanning Electron Microscopy and X-Ray Mapping
}

\author{
J. P. Neilly, A. D. Vogt and W. Dziki
}

Abbott Laboratories, Global Pharmaceutical Research and Development, Department R4R9, AP31, 200 Abbott Park Rd., Abbott Park, IL 60064-6202

Sticking is the phenomenon where pharmaceutical powders adhere to punch faces during tablet manufacturing. Sticking can accumulate over time resulting in removal of material from tablets, a tablet defect known as picking. Sticking and picking are common problems in tablet manufacture and much research has been dedicated to solving this problem $[1,2]$. This presentation will describe analysis of sticking residue to better understand the sticking mechanism.

A formulation known to have sticking problems consisted of an active pharmaceutical ingredient (API), microcrystalline cellulose (binder/diluent), colloidal silicon dioxide, (glidant), sodium croscarmellose (disintegrant), and magnesium stearate (lubricant) and was compressed on a Piccola lab scale tablet press. This formulation showed visible residue on punch faces starting at 500 compression cycles and severe sticking after 2000 compression cycles (Figure 1). Punches with sticking residue were initially studied directly on the tooling with a FEI XL30 environmental SEM, an Edax Genesis 4000 EDS and by an Spectral Dimensions Sapphire near infrared (NIR) chemical imaging system. In addition, hand pressed compacts were studied to characterize the response of the SEM and $\mathrm{x}$-ray mapping system to this formulation.

Punch faces were uniformly covered except for the center where the residue accumulated in greater quantities than the rest of the punch face (Figure 1). Elemental x-ray mapping of the compacts was able to detect four unique elements of the 5 formulation components (Figure 2). The fifth component (microcrystalline cellulose) could be identified by its moderately high oxygen levels and the absence of the other four unique elements. Maps of the punch face residue showed virtually no magnesium stearate, sodium croscarmellose or microcrystalline cellulose on the punch faces (Figure 3). Most of the residue was composed of API with low levels of silicon dioxide. NIR chemical imaging also showed the API to be a major component of the residue.

The results show SEM and x-ray mapping can identify and localize the components in the material adhering to the punch faces. These results, in conjunction with analysis of corresponding tablets with picking issues, can help identify formulations likely to have sticking issues in production and ultimately lead to a better understanding of why this phenomenon occurs.

\section{References}

[1] M. D. Tousey, Tablets and Capsules, October 2003

[2] F. Rowley, Pharmaceutical Technology Europe, Oct. 1, 2005 


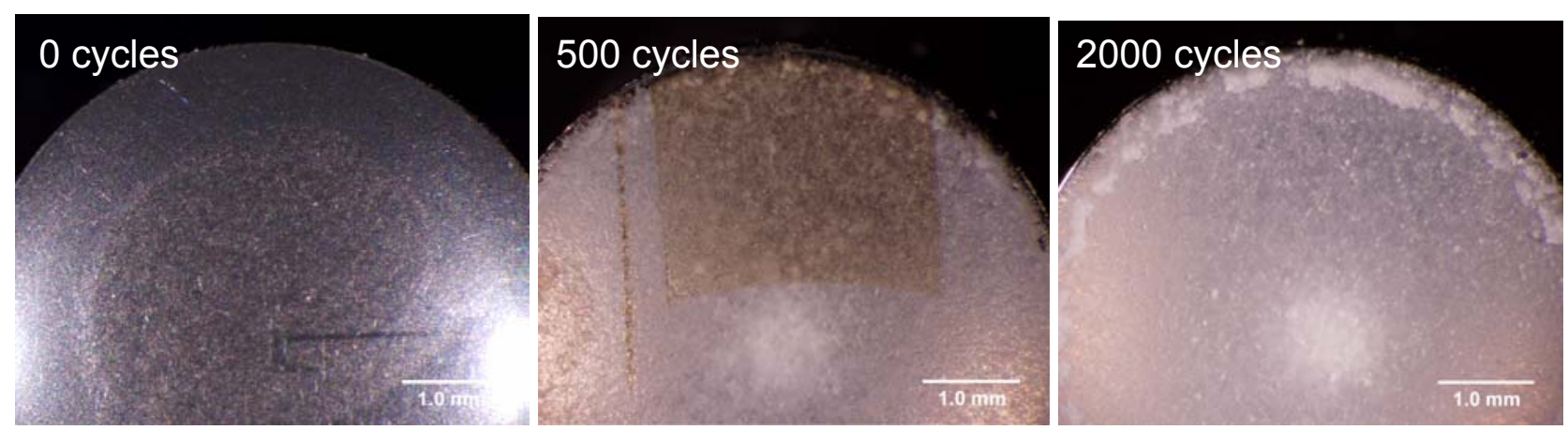

Figure 1. Upper tablet punch faces before use (left) after 500 compression cycles (center) and after 2000 compression cycles (right). Discolored square is result of x-ray mapping.
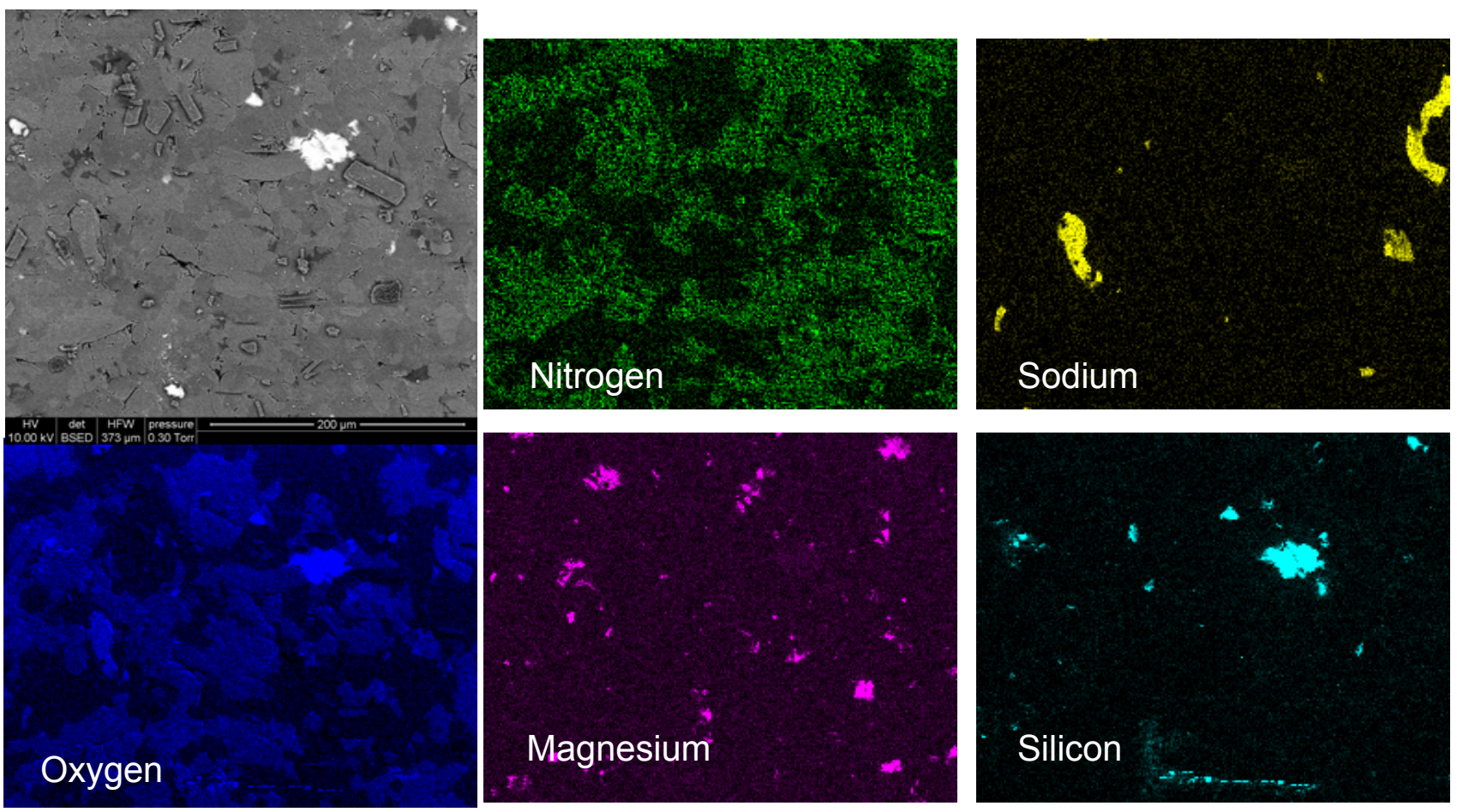

Figure 2. SEM image and x-ray maps of compact from formulation known to have sticking problems.
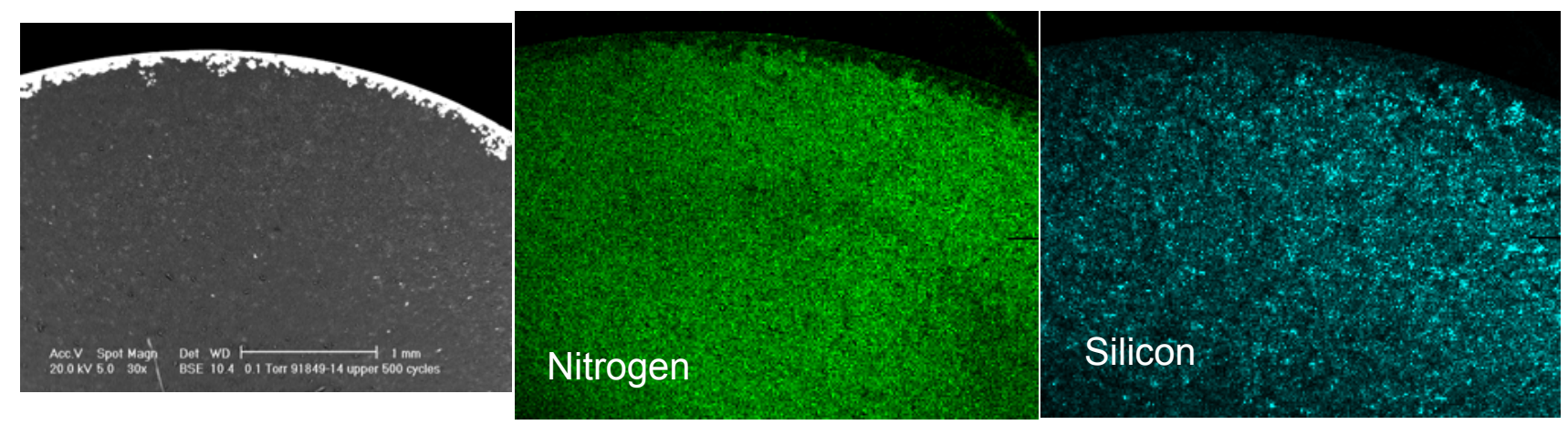

Figure 3. SEM image and x-ray maps of sticking residue on upper tablet punch face. 